Cahiers « Mondes anciens »

ANCIENS

Histoire et anthropologie des mondes anciens

5 | 2014

Maudire et mal dire : paroles menaçantes en Grèce ancienne

\title{
Ta mère ! Insulte et généalogie à la tribune démocratique
}

Noémie Villacèque

\section{CpenEdition}

Journals

Édition électronique

URL : http://journals.openedition.org/mondesanciens/1242

DOI : $10.4000 /$ mondesanciens. 1242

ISSN : 2107-0199

Éditeur

UMR 8210 Anthropologie et Histoire des Mondes Antiques

Référence électronique

Noémie Villacèque, "Ta mère ! Insulte et généalogie à la tribune démocratique », Cahiers " Mondes anciens » [En ligne], 5 | 2014, mis en ligne le 01 février 2014, consulté le 30 avril 2019. URL : http:// journals.openedition.org/mondesanciens/1242 ; DOI : 10.4000/mondesanciens.1242

Ce document a été généré automatiquement le 30 avril 2019

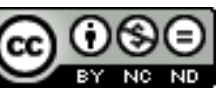

Les Cahiers «Mondes Anciens » sont mis à disposition selon les termes de la licence Creative Commons Attribution - Pas d'Utilisation Commerciale - Pas de Modification 4.0 International. 


\title{
Ta mère ! Insulte et généalogie à la tribune démocratique
}

\author{
Noémie Villacèque
}

De l'un il disait : « C'est un esclave, fils d'esclaves, qui devrait payer l'impôt du sixième, dans la classe des métèques ». D’un autre : « Il a des enfants

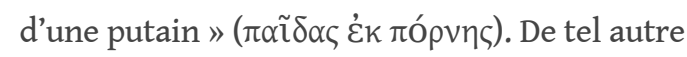

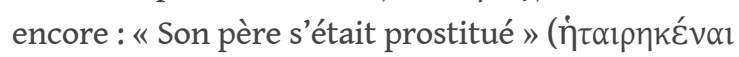

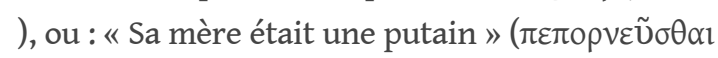

)...1

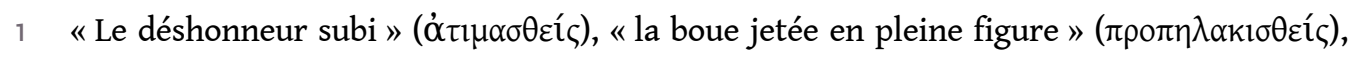

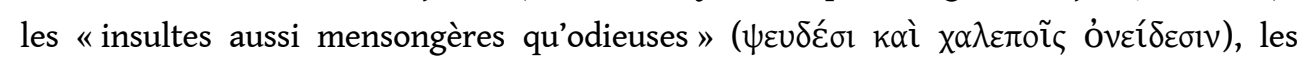

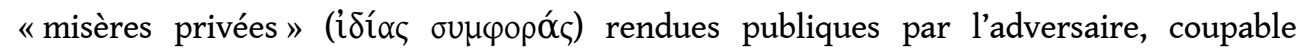

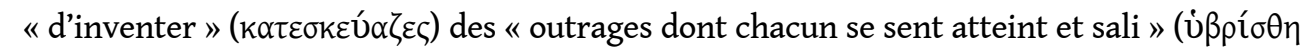

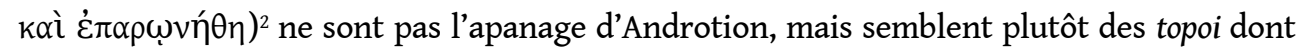
Démosthène lui-même était assez friand. Parmi ces multiples insultes, nous nous intéresserons, dans les pages qui suivent, à celles dont est accablée la mère de l'adversaire, toujours plus acerbes, toujours plus insidieuses ${ }^{3}$.

2 En 451/450 avant J.-C., " sur la proposition de Périclès ", rapporte Aristote, " on décida de ne pas laisser jouir de droits politiques quiconque ne serait pas né de deux citoyens $»^{4}$; cette loi, on le sait, ne fut pas sans conséquence pour les femmes, qui, en tant que mères, acquirent désormais un statut de premier ordre dans la vie civique. En effet, comme le dit Ion, dans la tragédie éponyme d'Euripide :

Et si j'avais un vœu à former, puissé-je être né d'une femme qui soit athénienne, afin d'avoir, par ma mère, droit de libre parole $(\pi \alpha \rho \rho \eta \sigma i ́ \alpha)^{5}$.

Il importe désormais d'être né non seulement d'un père citoyen, mais aussi d'une mère elle-même fille d'un citoyen et d'une fille de citoyen, elle-même fille d'un citoyen et d'une fille de citoyen, elle-même... : ainsi, pour reprendre les mots de Nicole Loraux (1990, p. 20), « la parenté ou, pour lui donner sa forme restreinte et absolument contraignante, la double filiation athénienne, constitue le soubassement de la citoyenneté, dont le 
mariage est la condition ». Aussi la mère de l'adversaire devint-elle pour les orateurs une cible de choix, car l'invectiver permettait de mettre en doute la citoyenneté de son fils et de le faire ainsi sortir de la communauté civique, de l'exclure du dèmos et, par conséquent, de contester son droit à la parole, de le priver de la parrhèsia consubstantielle à son statut de citoyen.

4 S'il ne devait pas être facile de contester la citoyenneté du père d'un individu, en revanche, la mère semble avoir constitué une cible plus aisée et donc plus fréquente ; son nom ne figurait sur aucun registre et sa participation aux rites réservés aux citoyennes, comme les Thesmophories par exemple, ne pouvait être attestée que par des témoins. Aussi le doute pesait-il toujours sur son statut et, comme l'a montré Robin Osborne (2010, p. 251-265), on se devait de revendiquer la bonne naissance de la femme, épouse, mère ou fille, sur les stèles funéraires, ultime moyen de reconnaissance sociale. L'emplacement et l'aspect de sa tombe étaient éloquents, si bien que, comme le dit Isée, il fallait que des

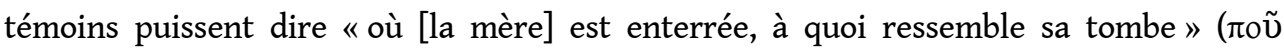

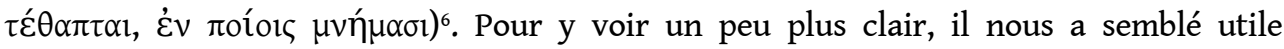
d'établir une typologie de l'invective dont les mères sont la cible.

En effet, si l'on examine de près les discours des orateurs, on distingue principalement trois types d'injures adressées aux mères: la mère étrangère, la mère prostituée et la mère indigente. Nous verrons comment ces injures, dont le point commun est de contester l'appartenance de l'adversaire au corps civique, deviennent pour ceux qui en sont la cible des éléments à charge qu'ils se doivent de réfuter.

6 La première façon d'insulter la mère de l'adversaire, la plus élémentaire, la plus simple, est de montrer qu'elle n'est pas fille de citoyen et/ou, du côté maternel, petite-fille de citoyen, mais qu'elle est étrangère à la cité voire de condition servile. On comprend dès lors l'importance de la citoyenneté - ou pas - du grand-père maternel, voire de l'arrière grand-père maternel. Car, Isée insiste sur ce point, « il ne suffit pas de dire le nom de la

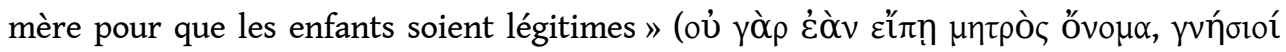
$\varepsilon \dot{i} \sigma \mathrm{lv})^{7}$. En effet, épigraphie et sources littéraires le confirment: si le patronyme (au génitif) fait partie de l'identité de l'individu, tant avant qu'après la loi de Périclès, le nom de la mère n'a jamais été convoqué pour y ajouter précision et légitimités ; quand, suite à l'accroissement de la population au $\mathrm{IV}^{\mathrm{e}}$ siècle, c'est devenu nécessaire, l'usage systématique du démotique a joué ce rôle?

7 Ainsi Eschine s'attache-t-il à démontrer, avec force détail - si bien qu'on est plus proche du psogos comique que de l'argumentation judiciaire - que Démosthène était « issu par [sa] mère des Scythes nomades ${ }^{10}$. Après avoir reconnu que « Démosthène eut pour père, car il ne faut pas mentir, un homme de condition libre, Démosthène de Péanie », il ajoute ceci : « du côté de sa mère et de son aïeul maternel, quelle est la situation ? je vais vous le dire ». Gylon du Céramique, un Athénien donc, aurait (entre 410 et 405 avant J.-C.) trahi la cité en livrant au roi du Bosphore cimmérien la forteresse de Nymphaion (dans la Crimée actuelle) dont il dirigeait la garnison athénienne. Il fut pour cela condamné à mort Eschine ne dit pas que la peine avait ensuite été commuée en amende. Il s'enfuit - ou plutôt il partit après s'être acquitté de son amende - pour « le Bosphore où règnent les tyrans ». Là, il fut très bien accueilli : le roi lui concéda un domaine et il put épouser une riche Scythe. Il eut d'elle deux filles à qui il donna de riches dots. Comme le note John Davies (1971, n 14625 II), Kèpoi (Les Jardins), la cité dont était originaire l'épouse de Gylon, était l'une des nombreuses colonies milésiennes des rives de la mer Noire, et ses filles, dont la mère de Démosthène, peuvent très bien avoir été grecques, sinon 
athéniennes. Bien entendu, ce n'est pas ce que veut laisser entendre Eschine : «l'une », avance-t-il perfidement, «se marie peu importe à qui, parce que je ne veux pas m'attirer la haine des gens ; l'autre à Démosthène de Péanie ». Ainsi, martèle Eschine, Démosthène,

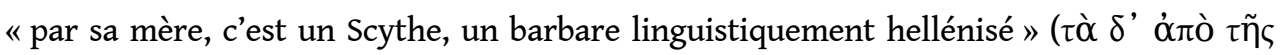

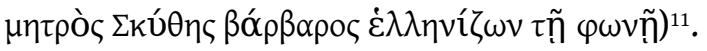

Il faut rappeler que la loi de Périclès avait été suspendue pendant la guerre du Péloponnèse, pour des raisons probablement démographiques, puis rétablie en 403 avant J.-C. Or on sait seulement que Cléoboulè, la mère de Démosthène, est née entre 408 et 400. Selon le raisonnement d'Eschine, si elle est née avant 403, Démosthène est citoyen ; si elle est née après 403, il ne l'est pas. Certes les adversaires de l'orateur le traitaient de "scythe ", de "bâtard", mais ils ne semblaient pas en mesure d'apporter la preuve de leurs allégations : aucune action pour usurpation du droit de cité ne semble avoir été intentée contre lui (Carlier 2006, p. 37).

Au-delà des interrogations que peut susciter cette généalogie de Démosthène, les accusations d'Eschine à l'encontre de la mère de son vieil ennemi sont intéressantes parce qu'elles font de lui « un nothos au sens politique du terme », pour reprendre la formule de Pierre Carlier (2006, p. 37), ou encore, pourrait-on dire, un double marginal.

Tout d'abord parce que Démosthène apparaît, par son ascendance maternelle, comme un Barbare - Eschine emploie le mot - quelqu'un qui parle grec, certes, mais qui, pour le reste, a tout d'un étranger : il est d'une famille riche et il est lié, tant par les accointances de son père que par le sang de sa mère, aux despotes orientaux.

11 Mais également parce que, à Athènes, « scythe » est non seulement un ethnonyme, mais aussi un nom commun, désignant les membres d'un corps d'archers composé d'esclaves publics. Ces derniers formaient, en effet, pour une grande partie du v $v^{e}$ et du IV siècle ${ }^{12}$, une force armée omniprésente dans la cité, assurant des tâches de maintien de l'ordre. Figure familière, l'archer scythe - presque une tautologie - s'installe durablement au cours du $v^{e}$ siècle dans l'imaginaire athénien comme le représentant par excellence de la marginalité, voire de la sauvagerie. Non seulement parce qu'il est une «brute » inculte, un «flic » qui ne comprend rien ni au grec ni aux subtilités de l'art tragique - c'est l'image qu'Aristophane en donne en 411, dans les Thesmophories ${ }^{13}$ - mais aussi et surtout parce qu'il manipule l'arc. Or, comme l'a montré François Lissarrague (1990, p. 125-127), l'arc est une arme marginale, une arme à la fois éphébique et barbare, une arme qui brouille la frontière entre la chasse et la guerre. L'archer scythe placé au centre de l'espace politique est un paradoxe vivant.

12 À en croire le chœur des Acharniens d'Aristophane, Thucydide, fils de Mélésias, vénérable combattant de Marathon déjà plus qu'octogénaire en 425 , a dû faire face, lors d'un procès,

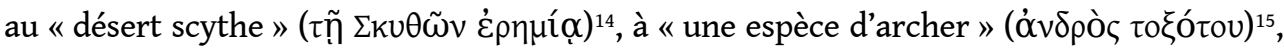
un orateur «jeune» (véov) et loquace ${ }^{16}$. Des citoyens jeunes - ou perçus comme tels dont l'activisme politique se déployait dans l'arène judiciaire, étaient donc comparés aux archers scythes. Pourquoi ? Parce que la mètis, la ruse habituellement associée à la jeunesse incitait à projeter sur ces talentueux orateurs la figure de guerrier irrégulier et déloyal la plus banale aux yeux des Athéniens de l'époque, celle des archers scythes. Le premier « chasseur noir » de ce nouveau genre dont on connaît le nom est sans doute Euathlos, ce jeune orateur qui s'en serait pris à Thucydide fils de Mélésias. Peut-on imaginer, à partir de là, que voie le jour un topos qui, potentiellement, fasse de tout orateur un archer, donc un Scythe? Quoi qu'il en soit, à partir de 451, pour contester la 
citoyenneté d'un individu, c'est sa mère qu'il faut attaquer. C'est probablement ce qui arrive à Euathlos lorsqu'Aristophane le traite d'archer scythe, tout en le désignant par un patronyme tout ce qu'il y a de plus athénien : « fils de Céphisodémos ${ }^{17}$.

En 343, plus de quatre-vingt ans après Euathlos, Démosthène est ainsi, du côté de sa mère, parfaitement étranger à ce dèmos auquel il prétend donner des leçons. C'est du moins le but de l'injure dont l'accable Eschine, et qui constitue un trait permanent du portrait qu'il brosse de son adversaire. On comprend mieux, dès lors, le sens du développement sur les origines de ce dernier: c'est parce qu'il est scythe que Démosthène est insidieux et fourbe, d'une façon inconnue aux Athéniens : «c'est pourquoi, en matière de perfidie, il

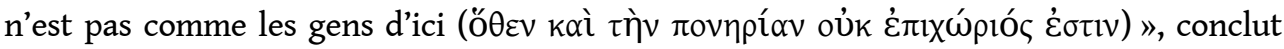
Eschine ${ }^{18}$.

Pour jeter le doute sur la citoyenneté de son adversaire, un orateur pouvait également choisir d'insulter sa mère de façon moins directe, mais tout aussi efficace. En effet, il lui suffisait d'insinuer que sa mère était une prostituée pour que l'adversaire en question soit considéré comme doublement bâtard (nothos) : sur le plan civique, parce que sa mère ne pouvait pas être une femme libre, épouse légitime d'un Athénien, mais était une esclave, et sur le plan ethnique, puisque le statut de prostituée semble aller de pair avec une origine étrangère. Cette insulte était courante à l'époque: on la trouve largement déployée dans la comédie, à l'encontre des génitrices des "démagogues ». La mère de Cléophon, par exemple, dont on ne manque pas de railler les prétendues origines thraces, est traitée de voleuse et de prostituée dans une comédie de Platon; celle d'Hyperbolos est qualifiée de prostituée, perse qui plus est, dans un fragment du Maricas d'Eupolis. D'ailleurs, si l'on en croit le coryphée des Nuées d'Aristophane, dans le Maricas, dont le titre est le sobriquet donné à Hyperbolos pour insinuer qu'il était d'origine barbare (Chantraine 2009, s. v. Mapikõ ; le surnom est peut-être d'origine iranienne), la mère du

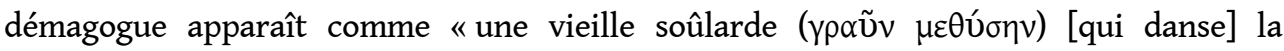
paillarde $»^{19}$. Le coryphée d'Aristophane remarque encore que «les autres [poètes comiques], depuis qu'un jour Hyperbolos leur a donné prise, ne cessent de le prendre

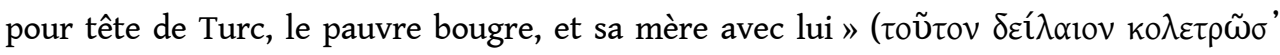

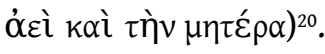

Isée reprend, à l'encontre de ses adversaires, ce vieux topos de la mère prostituée pour tâcher d'en démontrer la réalité :

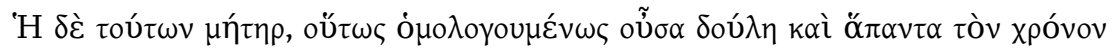

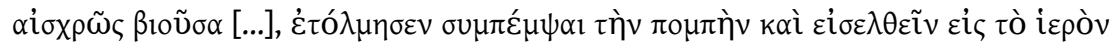

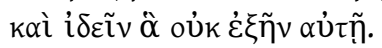

Mais la mère de ces gens, alors qu'elle était une esclave, au su de tous, et que sa conduite a toujours été scandaleuse [...] a eu l'audace d'accompagner la procession et de pénétrer dans le sanctuaire et de voir ce qui lui était interdit de voir ${ }^{21}$.

Dans ce passage, l'insulte n'a pas (ou pas seulement) pour objectif de déstabiliser l'adversaire sur le plan psychologique, comme on pourrait s'y attendre, mais répond, là aussi, à une stratégie argumentative visant à fragiliser le lien ténu qui lie la femme à la communauté civique et, partant, à contester la citoyenneté de ses enfants. En effet, la mère des adversaires n'est pas une citoyenne mais une esclave, menant une vie dissolue (

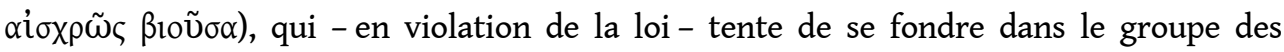
respectables Athéniennes en participant aux rites qui leur sont réservés. Seule la fraude maternelle a pu octroyer aux enfants - dont personne ne peut prétendre connaître le père - la qualité de citoyens. 
Démosthène ne se prive pas non plus d'utiliser cette insulte à l'encontre de la mère d'Eschine. Que ce dernier n'ait, à l'en croire, jamais joué autre chose que des troisièmes rôles n'est pas suffisant pour lui ${ }^{22}$, et il suggère les occupations peu recommandables de la mère de son adversaire ${ }^{23}$ :

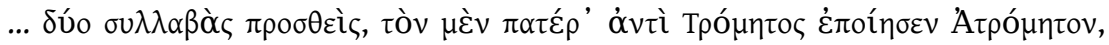

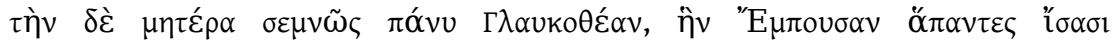

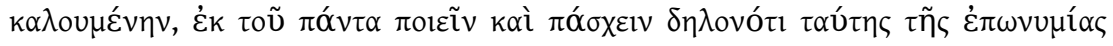

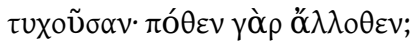

... ajoutant deux syllabes au nom de son père, il a fait de Tromès Atromètos et de sa mère tout à fait noblement Glaucothéa, quand chacun sait qu'on l'appelait Empousa et qu'elle avait reçu ce nom évidemment parce qu'elle faisait et subissait tout (quelle autre origine en effet?) ${ }^{24}$.

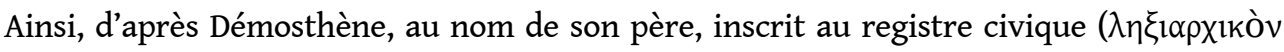

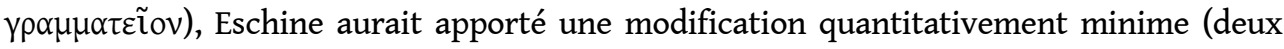

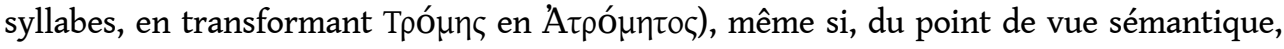

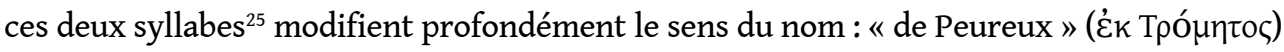
, le père d'Eschine serait devenu, par la magie de la composition, un "Intrépide " (Aтрó $\mu \eta \tau о \varsigma)$, les deux noms, Tromès et Atromètos étant par ailleurs mis en résonance grâce à la flexion. Ainsi, en se présentant, Eschine aurait déclaré s'appeler Aíoxívnc Ȧ tout d'abord, contrairement à son mari, elle ne serait connue que sous le surnom d'Empuse, que Démosthène a vite fait d'assimiler à un surnom professionnel, une sorte de «pseudo » de prostituée. Comme l'a souligné Stephen Usher $(1993, \text { ad loc. })^{26}$, ce surnom « renvoie à la magie et à l'illusion impliquant un changement de forme ». Les prostituées prenaient souvent des noms d'êtres surnaturels, comme Charybde ou Chimère. Empuse était un monstre aux ordres de la déesse des Enfers Hécate et capable de prendre « tantôt la forme d'un taureau, tantôt celle d'une mule, tantôt celle d'une femme d'une beauté exceptionnelle » (Aristophane, Grenouilles, 289-293). Démosthène suggère sans doute que Glaucothéa mimait cette figure mythique dans son activité professionnelle ou rituelle. Peut-être que sa spécialité était les jeux de rôles. Se débarrasser d'un tel surnom ne peut se faire par un simple tour de passe-passe grammatical ; il faut bien, d'après Démosthène, l'audace du fils d'Atromètos (et frère d'Aphobos) pour métamorphoser l'Empuse en chouette, l'emblème d'Athènes et d'Athéna, à travers le noble nom de Glaucothéa ${ }^{27}$. Mais Démosthène entend surtout démontrer, par cette digression, qu'Eschine, né esclave (

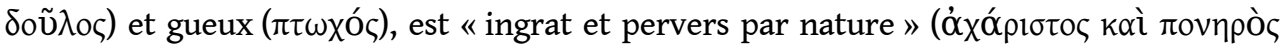
$\varphi$ ses origines obscures. Et s'il peut parader à la tribune, c'est grâce à sa mère et à l'argent de sa prostitution:

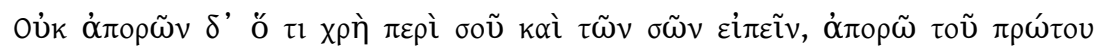

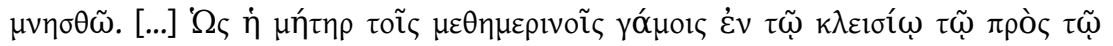

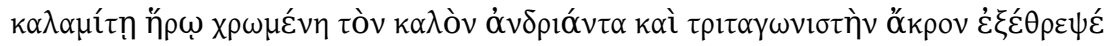

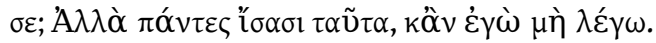

Pour ce que j'ai à dire de toi et des tiens, je n'ai que l'embarras du choix. [...] Que ta mère, grâce à ses mariages en plein jour dans la boutique voisine du héros Calamitès, a élevé la belle statue et l'excellent acteur de dernier ordre que tu es? Mais tout le monde le sait, même si je n'en parle pas ${ }^{29}$.

L'euphémisme et le paradoxe du mot "mariage» mis au pluriel pour désigner par sarcasme les rapports sexuels rémunérés, ainsi que l'oxymore formé par le syntagme $\mu \varepsilon \theta \eta \mu \varepsilon p ı v o i ̀ ~ \gamma \alpha ́$ นor «en plein jour », dans une société où l'union d'un homme et d'une 
femme se célébrait à la lueur des torches, sont certes des figures de style destinées à amplifier l'insulte. Cependant, ce repère temporel et le repère spatial mentionné dans la même phrase - la boutique voisine du héros Calamitès ${ }^{30}$ - sont aussi et surtout des détails circonstanciés qui transforment la simple insulte en accusation formelle. Or, insister sur le fait que la mère de son adversaire était une femme de mauvaise vie, qui se vendait dans des conditions dégradantes, en plein marché, au vu et au su de tous, permet encore de mettre doublement en doute sa citoyenneté : non seulement parce qu'une traînée ne saurait être une honnête Athénienne et ne peut être, au contraire, qu'une misérable étrangère, voire, comme le dit Isée, une esclave, mais aussi parce qu'une traînée ne peut donner une bonne éducation à son fils, parce qu'elle ne peut en faire qu'un " fils de pute "

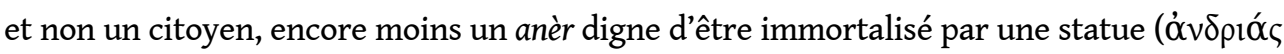
). Or Eschine ne s'est-il pas vendu à Philippe en acceptant, lors de l'ambassade, d'être son agent salarié, au mépris de l'intérêt de sa patrie ? N'a-t-il pas « loué » ses services ${ }^{31}$ ? En faisant cela, n'a-t-il pas suivi l'exemple de sa mère, lui qui accusait Timarque de se vendre? Dans son discours contre ce dernier, Eschine précise lui-même que le législateur " ne se demande pas comment l'acte [sexuel] a été commis. Non, quand il y a eu

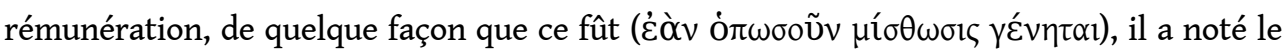
coupable d'infamie $»^{32}$.

Or Démosthène ne se contente pas de s'en prendre au «métier » de la mère d'Eschine, il insiste tout aussi lourdement sur le fait que ce dernier ait grandi dans la pauvreté, et nous allons voir que c'est là un troisième moyen pour rejeter l'adversaire hors de la communauté civique par le biais de sa mère. Mais laissons pour le moment Glaucothéa de côté, pour évoquer rapidement cette mère raillée non pas à la tribune mais au théâtre pour avoir été marchande d'herbes : la mère d'Euripide.

Le poète tragique est, en effet, brocardé à plusieurs reprises comme « fils de marchande d'herbes» (ó $\left.\tau \tilde{\eta} \varsigma ~ \lambda \alpha \chi \alpha v o \pi \omega \lambda \eta \tau \tilde{\alpha}^{\prime} \alpha \varsigma\right)^{33}$. De même qu'Eschine a été élevé entre la poussiéreuse salle de classe de son père et les douteuses initiations de sa mère, Euripide l'a été au milieu des herbes sauvages, cueillies et vendues par sa mère à la lisière de la terre cultivée, en marge de la cité, à en croire l'une des femmes réunies pour fêter les Thesmophories dans la pièce éponyme d'Aristophane. Les herbes sauvages (ó $\gamma p ı \alpha \lambda \alpha^{\prime} \chi \alpha v \alpha$ ), par opposition aux herbes de jardin, dont la culture présuppose un minimum de propriété foncière, contaminent ainsi de leur sauvagerie le jeune Euripide, qui devient lui-même, par glissement de sens, un "marginal » et un "sauvage", un homme qui ignore la civilisation au point de s'en prendre « sauvagement » aux femmes :

ö́p

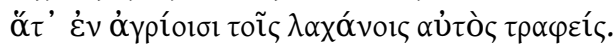

Il commet contre vous, femmes, des crimes sauvages,

car il a été lui-même élevé au milieu des herbes sauvages ${ }^{34}$.

Par-delà ce jeu de mots "anthropologique", outre le fait que les herbes sauvages devaient faire partie de l'ordinaire des plus pauvres et arriver en effet sur le marché grâce à $e^{35}{ }^{35}$, ce métier qu'aurait exercé la mère d'Euripide évoque, David Roselli (2005, p. 22) l'a très bien montré, "le monde honteux des classes inférieures qui peuplent le marché ", auquel sont associés les "nouveaux politiciens ». Les études à ce sujet sont nombreuses et je voudrais seulement rappeler ici que le Charcutier des Cavaliers est appelé Agoracritos «car c'est à l'agora, dans les disputes, qu'il fut nourri » ${ }^{36}$. Ainsi, «se moquer de la mère d'Euripide en la traitant de marchande d'herbes sauvages était une façon allégorique de parler de la popularité de ses drames auprès du dèmos. On dirait 
qu'Aristophane donne à voir à son public un poète issu de - et s'adressant à - la majorité du public théâtral, la classe laborieuse» (Roselli 2005, p. 28). Cet Euripide aristophanesque devient ainsi « une sorte de démagogue tragique» (Roselli 2005, p. 38). En même temps, la persona comique d'Euripide reçoit en héritage de sa marchande de mère le langage de la loidoria, qui est à la fois le langage du marché et la caractéristique la plus marquée de la Comédie ancienne. Ainsi, Aristophane fait d'Euripide un poète fondamentalement comique, un poète avec qui il peut se mesurer d'égal à égal (Saetta Cottone 2005, p. 298-303).

D’ailleurs, si elle raille les prétendues origines étrangères des démagogues, si elle traite leur mère de prostituée, la comédie n'hésite pas non plus à railler les petits métiers qu'exerçaient leurs parents avant eux. Présentés - le plus souvent à tort - comme étant issus d'un milieu modeste, Cléon, Hyperbolos, Cléophon et les autres, ces «singes amuseurs du peuple ", comme le dit le personnage d'Eschyle dans les Grenouilles ${ }^{37}$, sont collectivement traités d'ủ $\pi о \gamma \rho \alpha \mu \mu \alpha \varepsilon \varepsilon \tilde{l}^{38}$, comme si l'écriture était une activité dégradante, une tare ${ }^{39}$.

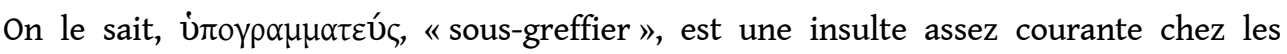
orateurs, si méprisante qu'elle est un quasi synonyme d'esclave. Ainsi Lysias peut-il écrire, à propos de Nicomachos : «d'esclave, il est devenu citoyen; de gueux, riche; de sous-greffier, législateur $»^{40}$. Or, cette insulte, Démosthène l'applique sans réserve à Eschine: pas moins de quatre occurrences dans le seul discours Sur l'Ambassade ${ }^{41}$, assorties de détails censés prouver que le reproche n'est pas à prendre au figuré : Eschine aurait réellement été le sous-greffier de divers magistrats, ainsi que du Conseil et de l'Assemblée. Faute de pouvoir s'appuyer sur l'opprobre social qui frappait les acteurs un siècle plus tôt, Démosthène veut montrer qu'Eschine est médiocre et besogneux et n'hésite pas à établir, par une cascade d'antithèses disposées en asyndète, un cruel parallèle entre sa propre carrière et celle d'Eschine :

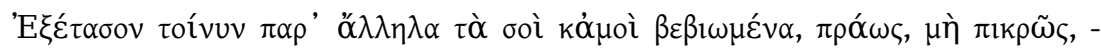

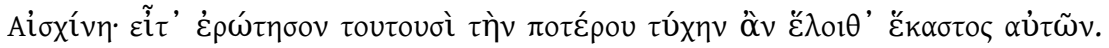

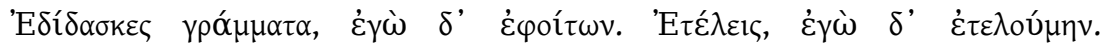

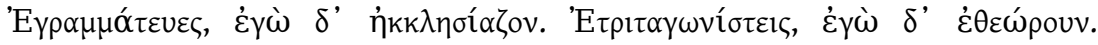

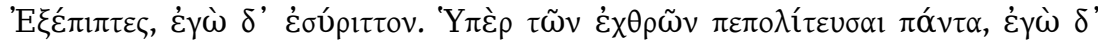

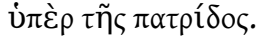

Examine donc l'une à côté de l'autre ton existence et la mienne, calmement, sans amertume, Eschine. Puis demande aux gens que voici quel est des deux sorts celui que chacun d'eux choisirait. Tu enseignais la lecture; moi, j'étudiais. Tu initiais; j'étais initié. Tu étais scribe; moi, je faisais partie de l'Assemblée. Tu jouais les troisièmes rôles; moi, j'assistais au spectacle. Tu tombais ; moi, je sifflais. Toute ta politique a été dans l'intérêt de l'ennemi; toute la mienne, dans l'intérêt de la patrie ${ }^{42}$.

On remarque, avec Harvey Yunis (2001, ad loc.), que dans l'inventaire comparatif de Démosthène, « comme dans l'invective elle-même, les activités attribuées à Eschine sont viles, serviles et méprisables. Les activités correspondantes attribuées à Démosthène présupposent certes contrôle et pouvoir mais, mis à part les connotations aristocratiques de la fréquentation des écoles, ce sont des activités que tout citoyen aurait pu exercer ", à commencer, bien sûr, par ceux qui composent le jury devant lequel l'orateur est en train de plaider. Démosthène le dit on ne peut plus clairement : «toi, d'ailleurs, tu n'as jamais servi à rien; moi, je faisais tout ce que doit faire un bon citoyen $»^{43}$. Ainsi l'invective

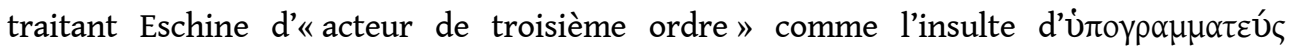


s'inscrivent-elles dans la stratégie rhétorique de Démosthène qui vise non seulement à disqualifier son adversaire en dévalorisant son milieu social - allant jusqu'à contester, au détour d'une incise, la régularité de son inscription au dème ${ }^{44}$ - et en lui déniant toute compétence professionnelle, mais, plus radicalement encore, à le disqualifier en tant que citoyen. Nécessiteux, Eschine ne peut penser qu'à ses intérêts privés et, pour s'enrichir, n'hésite pas à trahir la cité. Piètre acteur, sifflé jadis au théâtre, il n'a pas sa place à la tribune de l'Assemblée ou des tribunaux: il est, en fin de compte, indigne du statut de citoyen.

Or, comme les démagogues brocardés par la comédie, Eschine n'est pas tout à fait responsable de la médiocre situation qui est la sienne, ce sont ses parents qui sont en cause, et notamment sa mère :

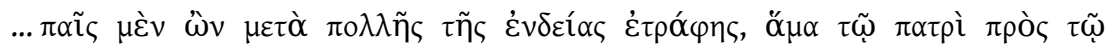

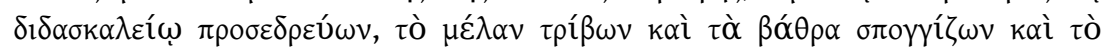

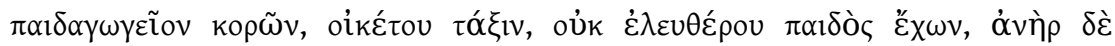

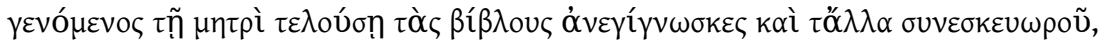

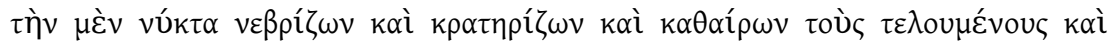

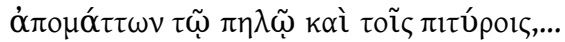

Dans ton enfance, tu as été élevé au milieu de la plus grande indigence, faisant, à côté de ton père, office d'auxiliaire dans une école, préparant l'encre, lavant les bancs, balayant la salle d'attente, ayant rang de serviteur et non pas d'enfant libre. Devenu homme, pendant que ta mère pratiquait les initiations, tu lui lisais les livres et tu collaborais à toutes ses machinations : toute la nuit tu présentais la peau de faon et le cratère, tu purifiais les initiés, tu les barbouillais de boue et de son... ${ }^{45}$

Démosthène s'attache à démontrer que son adversaire n'est pas sorti de cette indigence originelle, voire qu'il s'y est embourbé :

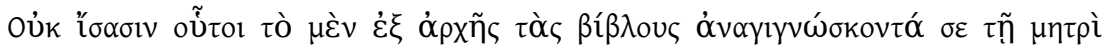

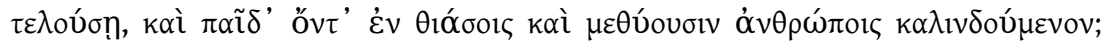

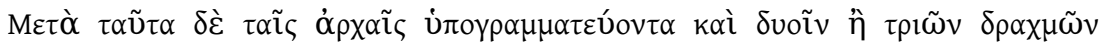

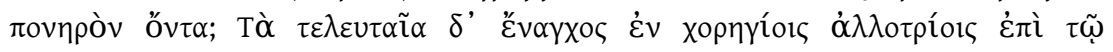
$\tau \rho \imath \tau \alpha \gamma \omega v l \sigma \tau \varepsilon \tilde{v} v \dot{\alpha} \gamma \alpha \pi \eta \tau \tilde{\omega} \varsigma \pi \alpha \rho \alpha \tau \rho \varepsilon \varphi o ́ \mu \varepsilon v o v ;$

Ces juges ne savent-ils pas que tout d'abord tu lisais les livres de ta mère pendant ses initiations et que, dans ton enfance, tu roulais dans des thiases d'ivrognes? qu'ensuite tu as été sous-greffier aux ordres des magistrats et que tu commettais des malhonnêtetés pour deux ou trois drachmes? enfin que tout récemment tu étais bien content de vivre en parasite en jouant les troisièmes rôles dans des représentations payées par d'autres ${ }^{46}$ ?

On remarque que, dans les deux passages que nous venons de citer, Démosthène demeure très vague quant à la nature des initiations pratiquées par la mère d'Eschine. Elles sont difficilement identifiables et diverses hypothèses ont ainsi pu être formulées: mystères de Dionysos (Cole 1980, p. 228, 236-237), mystères de Sabazios (Wankel 1976, p. 1133 sq. ; Burkert 1987, p. 19, 33, 43, 70, 96-97), rites orphiques (Parker 1983, p. 303), ou encore éleusiniens (Roussel 1930). Edward M. Harris, à qui nous devons ces références (1995, p. 25 et $n$. 24), explique que la diversité de ces interprétations est due au fait que Démosthène reste sciemment dans le flou pour mêler, non sans humour, les aspects exotiques de chacune de ces cérémonies (1995, p. 25). Or, grâce ces quelques zestes d'exotisme et à une goutte d'ivresse, Démosthène transforme les activités cultuelles de Glaucothéa-Empuse en une débauche scélérate. La mère d'Eschine, en effet, n'est-elle pas alors comme celle de Penthée, une de ces femmes qui « exaltent par leurs danses leur nouveau dieu, Dionysos, Bacchos, que sais-je ? Des cratères remplis de vin, dit-on, parmi leurs thiases se dressent. De tous côtés, les femmes vont à l'écart subir le bon plaisir des mâles $»^{47}$. Euripide fait 
ainsi de Penthée, roi de Thèbes, le porteur d'une vox populi qui n'est autre que celle des Athéniens assis sur les gradins du théâtre de Dionysos, majoritairement hostiles à tout ce qui, en matière de sacré, sort du cadre officiel. Or, si Euripide finit par mettre en pièces son Penthée et sa raison trop raisonneuse ${ }^{48}$, Démosthène se contente de jouer les Penthée, essayant à son tour de réveiller, chez ses auditeurs, la même méfiance. Il fait de Glaucothéa une Agavè de comédie qui, loin de servir, en femme et fille de citoyen, de respectables cultes civiques, s'adonne à des rites étrangers, autant dire à la luxure. Ainsi la pauvreté supposée des parents d'Eschine ${ }^{49}$, et notamment le trouble métier qu'exerçait sa mère, est pour son adversaire le moyen de démontrer sa profonde marginalité sociale pour le rejeter hors du corps civique.

Mais ce procédé n'est pas propre à Démosthène. Lui-même, dans un discours qu'il aurait écrit pour un client nommé Euxithéos et transmis sous le titre Contre Euboulidès, doit prendre les devants pour, cette fois, réfuter les accusations dont son client fait l'objet. Dans ce discours, le plaideur dit en effet aux jurés vouloir leur " prouver que [qu'il est] Athénien, et du côté paternel et du côté maternel, [leur] fournir en ce sens des témoignages [qu'ils reconnaîtront] véridiques, réfuter les diffamations et les calomnies » ${ }^{50}$. Et de justifier les métiers de marchande de rubans et de nourrice exercés par sa mère, en insistant sur le fait qu'" elle était Athénienne et citoyenne $»^{51}$. Cette insistance montre combien la seule évocation d'un petit métier suffit à jeter le doute sur la citoyenneté de celui et surtout de celle qui l'exerce, et par conséquent, sur sa descendance. Euxithéos le dit d'ailleurs on ne peut plus clairement :

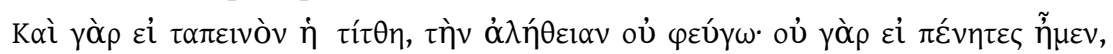

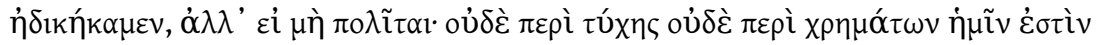

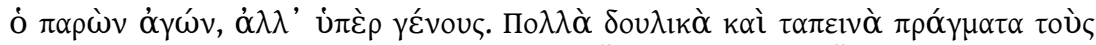

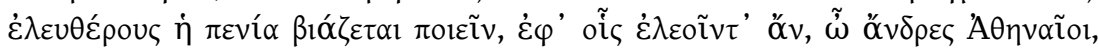

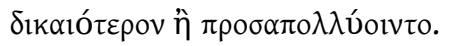

Que le métier de nourrice soit un petit métier, soit: la vérité ne me fait pas peur. Nous ne sommes pas coupables d'avoir été pauvres, nous le serions si nous n'étions pas citoyens. Il ne s'agit pas d'heureuse fortune ou d'argent dans ce procès, mais de descendance athénienne. La pauvreté contraint les personnes libres à faire bien des choses serviles et humiliantes : c'est une raison pour les plaindre, Athéniens, plutôt que pour les condamner par surcroît ${ }^{52}$.

Pour Claude Mossé (1999, p. 227), « le fait même qu'on ait pu utiliser l'argument du métier de sa mère contre Euxithéos montre bien qu'il s'agissait là de situations désespérées et que la norme demeurait pour la femme athénienne le travail domestique dans le cadre de l'oikos "; ainsi, le travail des épouses, filles et mères de citoyens hors de la maison aurait été très exceptionnel « et durant une période exceptionnelle aussi, celle des lendemains immédiats de la guerre du Péloponnèse et de la défaite ». David Cohen (1991, p. 149-153) souligne, au contraire, la nécessité pour les femmes de travailler non seulement pour se substituer aux hommes partis au combat ou pour faire face à une situation économique particulièrement difficile, mais aussi pour apporter des revenus supplémentaires, dans le cadre d'une économie de subsistance ; la multiplicité des occasions pour les femmes de sortir de chez elles l'amène ainsi à la conclusion qu'elles n'étaient pas confinées à l'intérieur de l'oikos. Mais l'étude systématique des sources, notamment épigraphiques, permet à Roger Brock (1994) d'aller plus loin encore et de démontrer que le travail des épouses des citoyens - nourrices, boulangères, pâtissières, blanchisseuses, accoucheuses voire médecins - n'avait rien d'inhabituel, même si elles étaient tenues à l'écart de certains métiers, tel que la vente de poissons ou les bains publics. 
31 Comment comprendre alors la charge dont Euxithéos fait l'objet, à travers sa mère, marchande de rubans et nourrice? David Cohen (1991, p. 135 sq.) l'explique, en s'appuyant sur les travaux de Pierre Bourdieu (1972), par une tension entre l'idéologie, où la norme de la femme recluse, confinée à l'intérieur de l'oikos, s'impose, et les pratiques sociales. Ainsi, bien que les épouses de citoyens aient toujours travaillé, l'idéal reste non seulement celui d'un genre masculin dominant (Just 1989, p. 113-125 notamment), mais aussi celui de l'élite, riche et oisive... D'ailleurs, un peu plus tôt dans le discours écrit pour lui par Démosthène, Euxithéos avait exhorté les jurés :

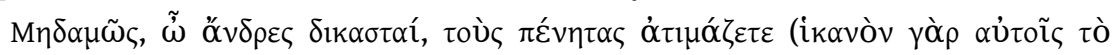

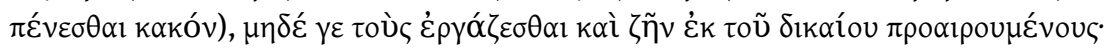

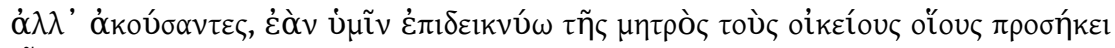

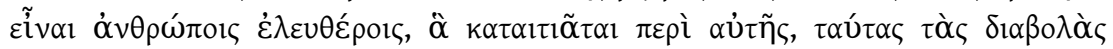

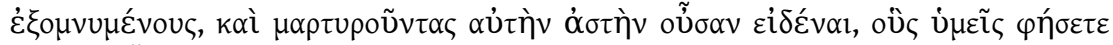

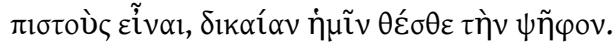

Non, juges, ne flétrissez pas les pauvres - la pauvreté est un malheur suffisant pour eux - ni ceux qui ont choisi de travailler et gagner leur vie par des moyens honnêtes. Écoutez-moi : et si je vous présente les parents de ma mère, des parents comme seules peuvent en avoir des personnes libres, opposant leurs serments aux calomnies de mon adversaire, si ces témoins que vous-mêmes déclarerez dignes de foi viennent attester qu'ils savent ma mère Athénienne, votez en notre faveur comme le veut la justice ${ }^{53}$.

32 Ainsi l'orateur doit-il, pour parer aux accusations de son adversaire, justifier non seulement le statut de tous les membres de la famille de sa mère (on se souvient des attaques d'Eschine à propos de la généalogie maternelle de Démosthène), mais plus encore : leur moralité. Insulter un orateur adverse en s'en prenant à ses origines sociales modestes, à la pauvreté de sa mère, au fait qu'elle ait été obligée de travailler pour vivre, devant un jury populaire, cela peut sembler surprenant. Comme l'explique Kenneth Dover (1974, p. 34-35), soit la plupart des jurés étaient aisés sans être riches et avaient davantage d'estime pour celui qui payait pour des services que pour celui qui les effectuait, soit ils n'étaient pas aisés - ce qui est, me semble-t-il, plus probable -, du moins pas dans leur majorité, mais ils appréciaient d'être traités comme tels. On connaît le passage du Contre Ergoclès de Lysias dans lequel l'orateur s'adresse à l'ensemble des jurés comme s'ils étaient tous de riches propriétaires accablés par les impôts ${ }^{54}$. L'appel du Contre Euboulidès à ne pas mépriser les pauvres et leurs petits métiers entre dans cette logique rhétorique. Quand Démosthène contre Eschine ou encore Euboulidès contre Euxithéos transforment l'insulte de la mère indigente en élément à charge, les mis en cause se doivent d'y répondre et de prouver, non pas que leur mère exerçait un métier plus honorable, mais que ce petit métier ne l'empêchait pas d'être fille de citoyen - et, par conséquent, qu'ils étaient eux-mêmes des citoyens à part entière.

On aura sûrement remarqué que ni Andocide ni Lysias ne sont cités dans ces pages. En fait, on ne trouve pas d'insulte relative à la mère dans les discours - assez peu nombreux, certes $-d u v^{e}$ siècle et même des premières décennies du IV ${ }^{e}$ siècle. Pendant toutes ces années, on les trouve dans la comédie, destinées à ceux qu'Eschyle qualifie, dans les Grenouilles, d'u் sociales, censées être moins prestigieuses que celles de la génération précédente. Indépendamment de l'historicité ou non de cette évolution sociologique, traiter la mère d'un adversaire de barbare et/ou de prostituée, c'était le disqualifier en tant que citoyen. Il semblerait que, dans le contexte social de la deuxième moitié du IV siècle, où les rapports entre classes étaient devenus extrêmement clivés, les orateurs se soient saisis de 
cet élément du psogos comique pour en tirer une série de reproches récurrents - la mère étrangère, la mère prostituée, la mère indigente - et pour les réinvestir en essayant, par des moyens rhétoriques, de les transformer en réels éléments à charge. Le procédé semble assez répandu pour que les parties se doivent de prendre les devants et de justifier des origines, des activités ainsi que de la moralité de leur mère, afin que ne soit pas mise en doute la légitimité de leurs revendications ou, plus radicalement, celle de leur présence à la tribune démocratique.

\section{BIBLIOGRAPHIE}

Bourdieu P. (1972), Esquisse d'une théorie de la pratique. Précédé de Trois études d'ethnologie kabyle, Genève.

Brock R. (1994), « The Labour of Women in Classical Athens », CQ 44 (2), p. 336-346.

Burkert W. (1987), Ancient Mystery Cults, Cambridge, Mass.

Carlier P. (2006), Démosthène, Paris.

Chantraine P. (2009), Dictionnaire étymologique de la langue grecque. Histoire des mots, Paris (première édition 1968).

Cohen D. (1991), Law, Sexuality, and Society. The Enforcement of Morals in Classical Athens, Cambridge, New York-Melbourne.

Cole S. G. (1980), « New Evidence for the Mysteries of Dionysus », GRBS 21, p. 223-238.

Couvenhes J.-Chr. (2012), « L'introduction des archers scythes, esclaves publics, à Athènes : la date et l'agent d'un transfert culturel ", dans Legras B. éd., Transferts culturels et droits dans le monde grec et hellénistique, Paris, p. 99-119.

Damet A. (2012), La septième porte. Les conflits familiaux dans l'Athènes classique, Paris.

Davies J. K. (1971), Athenian Propertied Families, 600-300 B.C., Oxford.

Debidour V.-H. (1965), Aristophane, Théâtre complet, vol. 1, Paris.

Debidour V.-H. (1999), Les Tragiques grecs. Eschyle, Sophocle, Euripide. Théâtre complet, Paris.

Detienne M. (1988), «L'espace de la publicité : ses opérateurs intellectuels dans la cité », dans Id. éd., Les savoirs de l'écriture en Grèce ancienne, Lille, p. 29-81.

Dodds E. R. (1943), Euripides Bacchae, Oxford.

Dover K. J. (1974), Greek Popular Morality in the Time of Plato and Aristotle, Berkeley.

Harris E. M. (1995), Aeschines and Athenian Politics, New York.

Hartog F. (1980), Le miroir d'Hérodote. Essai sur la représentation de l'autre, Paris.

Hunter V. (1990), « Gossip and the Politics of Reputation in Classical Athens », Phoenix 44 (4), p. 299-325.

Just R. (1989), Women in Athenian Law and Life, Londres-New York. 
Lissarrague F. (1990), L'autre guerrier. Archers, peltastes, cavaliers dans l'imagerie attique, Paris.

Loraux N. (1990), Les enfants d'Athéna. Idées athéniennes sur la citoyenneté et la division des sexes, Paris (première édition 1981).

Loraux N. (1993), « Aristophane, les femmes d'Athènes et le théâtre », dans Reverdin O. et Grange B. éd., Aristophane : sept exposés suivis de discussions, Entretiens sur l'Antiquité Classique 38, Genève, p. 203-253.

Loraux N. (1997), « Clisthène : diviseur-lieur d'Athènes », L'Inactuel 8, 1997, p. 5-26.

Mossé C. (1999), « Le travail des femmes dans l'Athènes de l'époque classique », Saitabi 49, p. 223-227.

Muller Ch. (2007), «Insaisissables Scythes : discours, territoire et ethnicité dans le Pont Nord », Pallas 73, p. 141-154.

Ober J. (2007), « Ability and Education. The Power of Persuasion », dans Carawan E. éd., The Attic Orators, Oxford-New York, p. 271-311 (= Ober J. [1989], Mass and Elite in Democratic Athens, Princeton, chapitre IV, p. 156-191).

Orfanos Ch. (2006), Les sauvageons d'Athènes ou la didactique du rire chez Aristophane, Paris.

Osborne R. (2010), Athens and Athenian Democracy, New York.

Parker R. (1983), Miasma. Pollution and Purification in Early Greek Religion, New York.

Roselli D. (2005), « Vegetable-Hawking Mom and Fortunate Son : Euripides, Tragic Style, and Reception », Phoenix 59, p. 1-49.

Roussel P. (1930), « Initiation préalable et les symboles éleusiniens », BCH 54, p. 58-65.

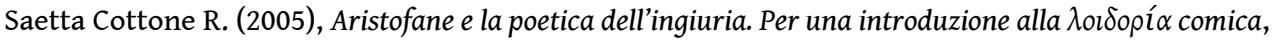
Rome.

Storey I. C. (2003), Eupolis. Poet of Old Comedy, Oxford-New York.

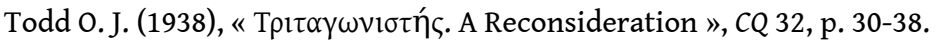

Todd S. (2007), « Lady Chatterley's Lover and the Attic Orators. The Social Composition of the Athenian Jury ", dans Carawan E. éd., The Attic Orators, Oxford-New York, p. 312-358 (= JHS 110, 1990, p. 146-170).

Usher S. (1993), Greek Orators V. Demosthenes, On the Crown (De Corona), Warminster.

Wankel H. (1976), Demosthenes. Rede für Ktesiphon über den Kranz, 2 vol., Heidelberg.

Yunis H. (2001), Demosthenes, On the Crown, Cambridge.

Zografou A. (2010), Chemins d'Hécate : portes, routes, carrefours et autres figures de l'entre-deux, Kernos Suppl. 24, Liège.

\section{NOTES}

1. Démosthène, Contre Androtion, 61. Sauf mention contraire, nous citerons les éditions et traductions de la C.U.F., éditions Les Belles Lettres.

2. Démosthène, Contre Androtion, 62-63 (traduction C.U.F. modifiée).

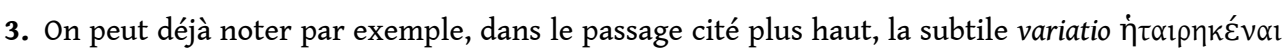
- $\pi \varepsilon \pi \circ \rho v \varepsilon \tilde{U} \sigma \theta \alpha \mathrm{l}$ : en effet, même si l'utilisation de deux termes différents sert, de prime abord, à 


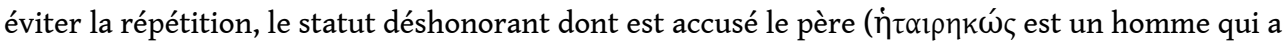
accepté, dans sa jeunesse, d'être le concubin d'un autre homme en échange d'avantages matériels, en dépit de la morale pédérastique) l'est moins que celui dont est accusée la mère, réduite à l'état de prostituée esclave ( $\pi$ ópvๆ).

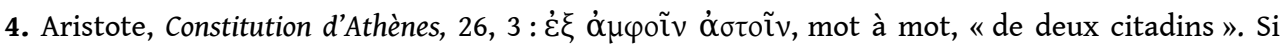
astos est souvent utilisé comme synonyme de politès, il a ici le mérite particulier d'éviter à Aristote l'utilisation juridiquement imprécise de politès pour une femme. Sur le contexte et les motivations de cette loi, voir la récente synthèse de Robin Osborne (2010, p. 244-251).

5. Euripide, Ion, 670-672 [traduction de V.-H. Debidour (1999) modifiée].

6. Isée, De la succession de Philoctémon, 64.

7. Ibid.

8. Si Isée, La succession de Pyrrhos, 14, 30-34, met en doute le statut d'une femme en tant qu'enfant légitime en arguant du nom dont son mari l'appelait, il ne s'agit pas, dans ce discours, de contester la légitimité d'un citoyen mais de contester le droit à un héritage que le mari de cette femme revendiquait au nom de son épouse (cf. Hunter 1990, p. 317-318; Damet 2012, p. 163).

9. Même si Aristote, Constitution d'Athènes, 21,5, fait remonter la généralisation du démotique à la réforme de Clisthène. Il s'agissait à l'époque de supplanter le patronyme dans sa fonction de marqueur d'appartenance à l'aristocratie, mais ce projet politique, si tant est qu'il existât, n'a jamais abouti ; voir Loraux 1997, p. 6-9 notamment.

10. Eschine, Sur l'Ambassade infidèle, 78 ; cf. 180.

11. Eschine, Contre Ctésiphon, 171-172. Nous avons modifié la traduction de la dernière phrase parce que nous pensons que $\dot{\varepsilon} \lambda \lambda \eta v i ́ \zeta \omega v \tau \tilde{n} \varphi \omega v \tilde{n}$ n'est pas un pléonasme (« qui parle grec dans son langage »), mais qu'il faut donner au participe le sens de « être acculturé, assimilé en milieu grec », avec la restriction induite par le datif modal « quant à sa langue ». Comme on le verra par la suite, d'autres traits de l'individu sont restés barbares.

12. Ailleurs, l'orateur parle de l'achat de ces esclaves comme d'un des exploits de la démocratie : Eschine, Sur l'Ambassade infidèle, 173,5. Sur le nomadisme scythe, voir Muller 2007; sur les Scythes à Athènes, voir Couvenhes 2012.

13. Aristophane, Thesmophories, 1001-1231.

14. Aristophane, Acharniens 704 (traduction Debidour 1965); cf. Orfanos, 2006, p. 172-173; Hartog 1980, p. 31-38.

15. Aristophane, Acharniens, 707 (traduction Debidour 1965).

16. Aristophane, Acharniens, 716-718.

17. Aristophane, Acharniens, 705.

18. Eschine, Contre Ctésiphon, 172 (nous traduisons).

19. Aristophane, Nuées, 555 (traduction Debidour 1965, modifiée).

20. Platon le Comique, fr. 61 ; 58 ; 60 K.-A. (Roselli 2005, p. 16 en particulier) ; Eupolis, fr. 9 K.A. (voir l'analyse du Maricas que propose Storey 2003, p.197-214; cf. Roselli 2005, p. 15); Aristophane, Nuées, 551-555.

21. Isée, La succession de Philoctémon, 49 (traduction C.U.F. modifiée).

22. D'après l'hypothèse d'O. Todd (1938, p. 36-38 notamment), Eschine était un très bon acteur qui a également joué des deuxièmes rôles ; mais Démosthène, jaloux de ses qualités physiques et vocales, refuse de le reconnaître et persiste à le traiter de tritagoniste.

23. Sur la profession des parents d'Eschine et le milieu social dans lequel l'orateur a été élevé, voir l'étude d'Edward M. Harris (1995, p. 21-29).

24. Démosthène, Sur la Couronne, 130.

25. Deux syllabes qui n'en sont qu'une dans la phrase de Démosthène: juste un $\alpha$ - privatif,

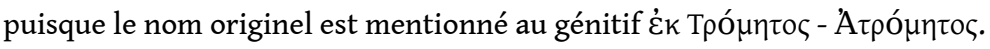

26. Sur Empuse en général, voir aussi récemment Zografou 2010, p. 227-233. 
27. Audace qu'il ne faut pourtant pas surestimer : Eschine, dans les quelques discours qui nous sont parvenus, ne désigne sa mère que comme la sœur de Cléobule, fils de Glaucos d'Acharnes, comme l'épouse d'Atromètos, ou tout simplement comme « ma mère ».

28. Démosthène, Sur la Couronne, 131.

29. Démosthène, Sur la Couronne, 129 (traduction C.U.F. légèrement modifiée).

30. Quel que soit le sens exact de l'allusion au «héros Calamitès ", il s'agit certainement d'un endroit connu de la plupart des Athéniens, très probablement un sanctuaire (Yunis 2001, ad loc.).

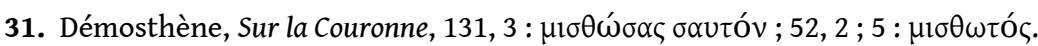

32. Eschine, Contre Timarque, 165.

33. Aristophane, Thesmophories, 387. Voir également Thesmophories, 455-456; Acharniens, 473-479; Grenouilles, 840.

34. Aristophane, Thesmophories, 455-456. Cf. Loraux 1993, notamment p. 233.

35. Les $\dot{\alpha} \gamma \rho ı \lambda \alpha_{\chi} \chi \alpha v \alpha$ ou $\chi 0 ́ \rho \tau \alpha$ - chicorée sauvage, pissenlit, pourpier, ou encore laiteron maraîcher - sont encore très importants dans l'alimentation des Grecs modernes, cueillis et vendus sur les marchés ou de porte à porte, essentiellement par des femmes.

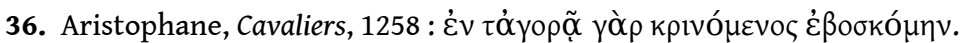

37. Aristophane, Grenouilles, 1085.

38. Aristophane, Grenouilles, 1083-1088.

39. Sur l'importance politique de la "chose écrite» et de ses techniciens aux débuts de la démocratie, ainsi que sur les états d'âme de ses adversaires, voir l'analyse de Marcel Detienne (1988, p. 64-72 en particulier).

40. Lysias, Contre Nicomachos, 27.

41. Démosthène, Sur l'Ambassade, $70 ; 200 ; 237 ; 249$.

42. Démosthène, Sur la Couronne, 265. Voir, plus largement, 257-264. Sur le «manque d'éducation » que Démosthène reproche à Eschine, voir Ober 2007, p. 301-304.

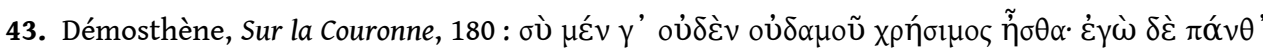

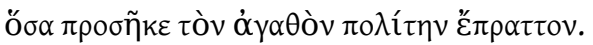

44. Démosthène, Sur la Couronne, 261 : « tu fus inscrit au registre de ton dème, tant bien que mal ». Contester la régularité de l'inscription de quelqu'un à son dème, c'est contester sa qualité de citoyen. Mais l'insinuation reste sans suite.

45. Démosthène, Sur la couronne, 258-259. Pour une discussion sur la plausibilité de ces allégations, voir Harris 1995, p. 29-30. Sur les « valeurs sociales » mises en avant par les orateurs, et par conséquent, sur leurs attaques mutuelles, voir Todd 2007, p. 164-167.

46. Démosthène, Sur l'Ambassade, 199-200 (traduction C.U.F. légèrement modifiée).

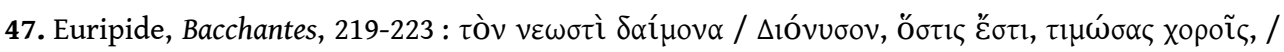

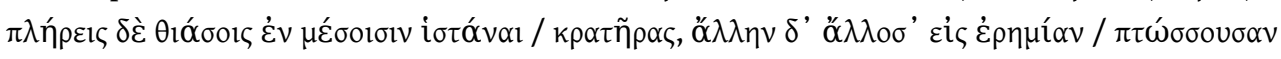

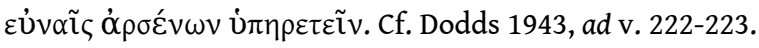

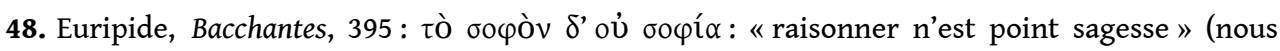
traduisons).

49. En réalité, si elle n'appartenait certes pas à l'aristocratie, la famille d'Eschine n'était pas indigente, loin de là : dans son étude, Edward M. Harris (1995, p. 26-29) montre que les membres de la famille ont servi comme hoplites et que le propre frère de l'orateur, Philocharès, a été élu stratège à trois reprises.

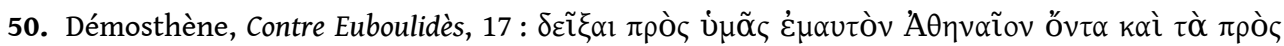

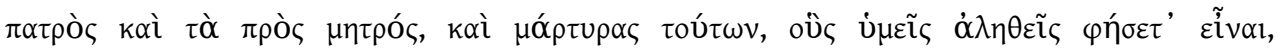

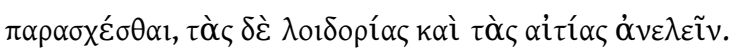

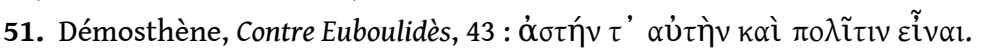

52. Démosthène, Contre Euboulidès, 45.

53. Démosthène, Contre Euboulidès, 36. 
54. Lysias, Contre Ergoclès, 3 : «Il serait étrange, en effet, de vous voir aujourd'hui, accablés comme vous l'êtes par les impositions, pardonner à la corruption et au vol... ».

\section{RÉSUMÉS}

Avec la loi de Périclès de 451/450 et la soudaine apparition de la matrilinéarité dans le droit athénien, les femmes acquirent une place importante dans la vie civique. À l'assemblée comme dans les tribunaux, un des procédés pour combattre un adversaire fut désormais de s'en prendre à sa mère. L'étude du corpus des orateurs permet de distinguer trois types d'injures la concernant : la mère étrangère, la mère prostituée et la mère indigente. Ces injures, qui semblent trouver leur source dans le psogos comique, devinrent pour ceux qui en étaient la cible des éléments à charge qu'ils se devaient de réfuter. En effet, mettre en doute la naissance, les mœurs et/ou le statut social de la mère revenait à contester la citoyenneté de son fils, l'exclure du dèmos et le priver de toute crédibilité, voire de son droit à la parole.

As a consequence of Pericles' citizenship law of 451/450, and the sudden arrival of matrilineality in Athenian law, women gained an important role in Athenian civic life. Henceforth, in the Assembly or in the law courts, one of the simplest methods for dealing with an adversary was to insult his mother. Study of the rhetorical corpus allows three kinds of insults, targeting the adversary's mother, to be distinguished: she could be described as a foreigner, a prostitute or a pauper. Seemingly derived from the comic psogos, abusive rhetoric became a major issue for litigants and something which had to be refuted, since casting doubt on the mother's birth, morality and/or social status amounted to contesting the son's citizenship, excluding him from the demos, depriving him of any credibility and even of his parrhesia.

\section{INDEX}

Mots-clés : mère, femme, démocratie, insulte, psogos, comédie, orateur, citoyenneté

Keywords : mother, woman, democracy, abuse, psogos, comedy, orator, citizenship

\section{AUTEUR}

\section{NOÉMIE VILLACÈQUE}

Université de Reims Champagne-Ardenne, Centre d'Études et de Recherche en Histoire Culturelle (CERHIC - EA2616) 